\title{
Análisis epidemiológico de la mortalidad por enfermedad pulmonar obstructiva crónica en el estado de Yucatán en 2017
}

\author{
Epidemiological analysis of chronic obstructive pulmonary \\ disease mortality in the state of Yucatan in 2017
}

\author{
Eduardo Briceño-Souza, ${ }^{*}$ Eduardo Falcón-Solís, ${ }^{*}$ Claudina Vázquez-Madariaga,* \\ Eduardo Vidal-Rosado, ${ }^{*}$ Nina Méndez-Domínguez*
}

*Universidad Marista de Mérida, Yucatán, México.

\begin{abstract}
RESUMEN. Introducción: La enfermedad pulmonar obstructiva crónica es una enfermedad que actualmente representa la cuarta causa de muerte en el mundo y en la población mexicana es una enfermedad que tiene alta tasa de mortalidad. De acuerdo con el reporte PLATINO y el criterio GOLD, la prevalencia es de $7.8 \%$ en personas mayores de 40 años. Los pacientes con enfermedad pulmonar obstructiva crónica mueren por exacerbaciones y complicaciones de la propia enfermedad. En nuestro entorno entre 50 y $80 \%$ muere fundamentalmente por agudización del padecimiento, en 8.5-27\% por cáncer de pulmón o por otras causas relacionadas. Material y métodos: Es un estudio epidemiológico observacional de tipo analítico con corte transversal retrospectivo, se incluyeron los registros de defunciones en adultos que se encuentran en las bases de datos de la Dirección General de Información en Salud (México), Secretaría de Salud registrados en el año 2017. Como variable dependiente se utilizó entidad de ocurrencia y como variables independientes se utilizaron el sexo, estación de ocurrencia, edad y el antecedente de asistencia médica. Resultados: En 2017 se registraron 22,954 muertes por enfermedad pulmonar obstructiva crónica en sus diversas modalidades para ambos sexos a nivel nacional, de los cuales 307 se dieron en el estado de Yucatán, México. La tasa de mortalidad por enfermedad pulmonar obstructiva crónica a nivel nacional se observó de 27.3 por cada 100,000 habitantes y en Yucatán fue de 20.2 por cada 100,000 habitantes. Por medio de la prueba t de Student se pudo observar una diferencia significativa $(p=0.000)$ en la edad de muertes por enfermedad pulmonar obstructiva crónica en hombres y mujeres, en mujeres representa una media de 80.4 años de edad y en hombres de 79.06 años. El porcentaje de personas que recibió asistencia médica a nivel nacional fue de $92.37 \%$, mientras que en el estado de Yucatán fue de $88.15 \%$. Conclusión: Existe una relación directa entre el sexo y el número de muertes por enfermedad pulmonar obstructiva crónica, así como la edad de fallecimiento de los pacientes. Se demostró que en Yucatán el número de casos disminuye durante el invierno y que la asistencia médica es menor en comparación con la de nivel nacional.
\end{abstract}

Palabras clave: Enfermedad pulmonar obstructiva crónica, epidemiología EPOC, mortalidad EPOC, infección respiratoria.

\begin{abstract}
Introduction: Chronic obstructive pulmonary disease (COPD) is a disease that currently represents the fourth cause of death in the world and in the Mexican population is a disease that has a high mortality rate. According to the PLATINO report and the GOLD criteria, the prevalence is $7.8 \%$ in people over 40 years of age. Patients with COPD die from the disease itself, in our environment between $50-80 \%$ die mainly from exacerbation of the disease, in $8.5-27 \%$ due to lung cancer or other related causes. Material and methods: This is an analytical observational epidemiological study, with a retrospective cross-section, including the death records in adults that are found in the databases of the General Directorate of Health Information (Mexico), Ministry of Health, registered in the year 2017. As a dependent variable, the occurrence entity was used and as independent variables gender, season of occurrence, age and medical assistance were used. Results: In 2017, there were 22,954 deaths due to COPD in its various modalities for both sexes nationwide, of which 307 occurred in the state of Yucatán. The mortality rate from COPD at the national level was observed at 27.3 per 100,000 inhabitants and in Yucatan it was 20.2 per 100,000 inhabitants. By means of the Student's t-test, a significant difference $(p=0.000)$ in the age of deaths due to COPD in men and women could be observed, representing in women the average of 80.4 years of age and in men of 79.06 years of age. The percentage of people who received medical assistance at the national level of $92.37 \%$ while in the state of Yucatan was $88.15 \%$. Conclusion: There is a direct relationship between gender and the number of deaths due to COPD, as well as the age of death of patients; It was shown that in Yucatan the number of cases decreases during the winter and that medical assistance is lower compared to the national level.
\end{abstract}

Keywords: Chronic obstructive pulmonary disease, respiratory infection, COPD mortality, COPD epidemiology.

Correspondencia:

Dr. Eduardo Briceño-Souza

Universidad Marista de Mérida,

Campus Ciencias de la Salud. Yucatán, México.

Correo electrónico: eduardobrices@gmail.com

Trabajo recibido: 03-IX-2019; aceptado: 17-III-2020. 


\section{INTRODUCCIÓN}

La enfermedad pulmonar obstructiva crónica (EPOC) es una enfermedad caracterizada por la limitación del flujo de aire, usualmente es progresiva y se asocia con una respuesta inflamatoria anormal de los pulmones ante partículas o gases nocivos. El manejo y tratamiento está encaminado a la mejoría de la sintomatología en lugar de una remisión de la entidad. ${ }^{1,2}$

De acuerdo con el reporte PLATINO y el criterio GOLD la prevalencia es de $7.8 \%$ en personas mayores de 40 años; asimismo, se ha observado que se presenta con más frecuencia en hombres $11 \%$ en comparación con las mujeres 5.6\%. Esta enfermedad se incrementa con la edad, en personas mayores de 60 años se incrementa hasta en $18.4 \%$ y en personas entre 50 y 59 años se manifiesta en 4.5\%. Existe una relación directa entre la EPOC y el número de cajetillas de cigarrillos fumados, el riesgo de padecerla incrementa si existe un consumo mayor de 10 cajetillas al año en $15.7 \%$ y en las personas con un consumo menor de 10 cajetillas el riesgo disminuye a $6.3 \% .^{3,4}$

La EPOC es una enfermedad que se manifiesta con más frecuencia en la población masculina, la población femenina se ve más afectada no sólo por el consumo del tabaco, sino por la exposición crónica a humo de biomasa; aproximadamente $\mathbf{1 4 . 5}$ millones utilizan la leña para cocinar, los estados del sur del país (entre ellos Yucatán) son los más expuestos. El Instituto Nacional de Enfermedades Respiratorias Ismael Cosío Villegas (INER) de la Ciudad de México señala que 30\% de las personas que padecen EPOC se debe a la exposición al humo de leña y de éstas $88 \%$ son mujeres. ${ }^{4,5}$

Los datos más recientes han demostrado que la mayor parte de las personas con una historia significativa de consumo de cigarrillos tiene tendencia a desarrollar una obstrucción del flujo aéreo. ${ }^{1}$ Un medio ambiente contaminado y la exposición ocupacional a polvos y químicos son factores importantes que aunados al tabaquismo pueden influenciar el consecuente desarrollo de la enfermedad. ${ }^{5,6}$

La EPOC en la actualidad representa la cuarta causa de muerte en el mundo, aunque es altamente prevenible, las tasas de mortalidad en 2015 registradas por la Organización Mundial de la Salud (OMS) fueron de 3.17 millones y los costos de ésta se han ido incrementando; se ha evidenciado que es superior a la de la población general, puesto que los pacientes con EPOC suelen tener otras comorbilidades asociadas. En la población mexicana es una enfermedad que tiene alta tasa de mortalidad; sin embargo, no se le ha dado el interés correspondiente y en muchas ocasiones es un padecimiento infradiagnosticado., ${ }^{1,2}$

Los pacientes con EPOC mueren por la propia enfermedad, en nuestro entorno entre 50 y $80 \%$ fallecen fundamentalmente por agudización del padecimiento, en $8.5-27 \%$ por cáncer de pulmón o por otras causas relacionadas. En etapas más tempranas la mayoría de las muertes son de origen no respiratorio; y se le puede atribuir a cáncer de distinto origen y enfermedades vasculares como cardiopatía isquémica y accidente vasculocerebral. Sin embargo, para evitar la variabilidad en el registro de las causas específicas de muerte, la mayor parte de los estudios utilizan la mortalidad global como variable dependiente porque la mayoría de las causas de muerte son debidas a la EPOC.?

El índice de mortalidad se presenta más en varones de edad avanzada en temporada de invierno, cuando las temperaturas son más bajas y los factores de exacerbación son más prevalentes. ${ }^{8}$

La mortalidad de EPOC se relaciona directamente con su gravedad, durante mucho tiempo se ha considerado el volumen espiratorio forzado en el primer segundo (FEV1) como el predictor más importante de mortalidad. Se ha encontrado que la espirometría no discrimina entre los estadíos de enfermedad, entre crónica y agudizada, por lo cual se plantean en la investigación factores asociados que agraven o incrementen la probabilidad de la mortalidad en estos pacientes como comorbilidades asociadas, sexo masculino y lugar de residencia. De igual manera se propone identificar si la tasa de mortalidad está asociada con los meses de invierno. ${ }^{6-8}$

Dado que es una enfermedad cuyo manejo y tratamiento se basa en la remisión de los síntomas, la prevención del tabaquismo así como el cese de su consumo y menor exposición a factores de exacerbación son medidas por demás importantes, pues se ha evidenciado que la disminución de estos factores es la única intervención que retrasa la pérdida de la función pulmonar.,

\section{Objetivo}

El objetivo de este artículo es analizar, comparar y correlacionar la epidemiología de la EPOC en el estado de Yucatán, México, con las cifras obtenidas a nivel nacional en las variables de edad, sexo, estación de ocurrencia y asistencia médica recibida.

\section{MATERIAL Y MÉTODOS}

La fuente de los datos aquí presentados deriva de los registros de mortalidad, procedentes de los certificados de defunción. Todos los diagnósticos contenidos en las bases de datos son por norma corroborados y registrados con fines estadísticos mediante la Dirección General de Epidemiología y proporcionados desde las bases de datos abiertos del Instituto Nacional de Estadística y Geografía (INEGI) y codificados de acuerdo con la Clasificación Estadística Internacional de Enfermedades y Problemas Relacionadas con la Salud en su décima edición (CIE-10). 
Tabla 1: Total de muertes por EPOC en el año 2017, con distribución a nivel nacional y estado de Yucatán por sexos.

\begin{tabular}{|l|r|r|r|}
\hline & Hombres & Mujeres & \multicolumn{1}{c|}{ Total } \\
\hline Nacional & 11,184 & 11,140 & 22,954 \\
\hline Yucatán & 157 & 150 & 307 \\
\hline & 11,341 & 11,290 & 23,261 \\
\hline
\end{tabular}

EPOC $=$ enfermedad pulmonar obstructiva crónica.

En el presente estudio se incluyeron mediante filtrado, los registros de mortalidad correspondientes a los diagnósticos CIE-10 J440, J441, J448 y J 449 correspondientes respectivamente a EPOC, con infección aguda de las vías respiratorias inferiores; EPOC por exacerbación aguda no especificada; otras EPOC especificadas, y EPOC no especificada.

Como variable dependiente se definió la entidad de ocurrencia, categorizando al estado de Yucatán en contraste con la población nacional con la finalidad de compararlo con las diferentes entidades federativas.

Para efectos de este estudio se incluyeron las siguientes variables independientes:

- Sexo: condición que distingue el género entre hombres (masculino) y mujer (femenino).

- Estación de ocurrencia: tiempo, temporada en el que se divide el año calendárico cuando ocurren las defunciones.

- Edad: número de años de vida que ha cumplido al momento que se presenta la muerte.

- Asistencia médica: presencia de servicios de salud relacionados con la enfermedad previo a la muerte.

Los procesos de codificado y análisis estacional expuestos corresponden a los descritos con respecto a la mortalidad nacional y en Yucatán por estación y a causa de enfermedades transmisibles y no transmisibles. ${ }^{9-11}$

El procesamiento de datos se realizó mediante el programa STATA 14 para codificar datos, para efectos del estudio se clasificaron las siguientes variables: entidad de ocurrencia distribuida en estados de la República Mexicana, edad y sexo, la estación de ocurrencia de acuerdo al año 2017 y el lugar de ocurrencia del evento en cuanto a un medio hospitalario u extrahospitalario. Para describir la estadística se usaron medidas de tendencia central y de dispersión. Se usó la prueba de $\chi^{2}$ y t-Student para la estimación de las variables con un intervalo de confianza al 95\% estableciendo un valor de $\mathrm{p}<0.05$ para representar un valor estadísticamente significativo. Se utilizó una comparación de hipótesis para conocer la proporción de defunciones por EPOC en el estado de Yucatán con la proporción a nivel nacional (todos los estados menos Yucatán); de igual manera en las estaciones del año con mayor ocurrencia de acuerdo con el sexo del fallecido.

\section{RESULTADOS}

Con base en los registros oficiales, durante 2017 se registraron 22,954 muertes por EPOC en sus diversas modalidades para ambos sexos a nivel nacional, de los cuales 307 se dieron en el estado de Yucatán. En la Tabla 1 se desglosan los fallecimientos por EPOC en el estado de Yucatán en hombres y mujeres.

La tasa de mortalidad por EPOC a nivel nacional se observó de 27.3 por cada 100,000 habitantes, mientras que en el estado de Yucatán fue de 20.2 por cada 100,000 habitantes como se muestra en la siguiente tabla (Tabla 2).

Con respecto a la proporción de muertos según el sexo se detectó que en el estado de Yucatán 48.85\% de las muertes son correspondientes al sexo femenino, en tanto que el sexo masculino representó $51.14 \%$ de las muertes.

En cuanto a la proporción de muertes por EPOC según la estación de ocurrencia se halló que en el estado de Yucatán la estación con mayor número de casos fue verano con $29.3 \%$, seguido de primavera con $24.1 \%$, invierno con $23.4 \%$ y por último otoño con $23.1 \%$; mientras que a nivel nacional se detectó el mayor número de casos en invierno con $29.3 \%$, seguido de primavera con $27.5 \%$, otoño con $23.1 \%$ y verano con $20.3 \%$ como se muestra en las Figuras 1 y 2.

Por medio de la prueba t de Student se pudo observar una diferencia significativa $(p<0.001)$ en la edad de muertes por EPOC en hombres y mujeres, en mujeres representó una media de 80.4 años de edad y en hombres de 79.1 años, con lo que se puede inferir que los hombres tuvieron una mortalidad a edades más tempranas que las mujeres.

Mediante un análisis de regresión se identificó que los hombres fueron más propensos a fallecer a causa de EPOC, mostrando una razón de riesgo de 1.37 a 1.00 con valor de $<0.01$.

Tabla 2: Tasa de mortalidad por EPOC en el año 2017.

\begin{tabular}{|l|c|c|}
\hline & Nacional & Yucatán \\
\hline $\begin{array}{l}\text { Muertes por } \\
\text { EPOC }\end{array}$ & 22,954 & 307 \\
\hline $\begin{array}{l}\text { Población mayor } \\
\text { de } 18 \text { años }\end{array}$ & $84,070,223$ & $1,517,290$ \\
\hline $\begin{array}{l}\text { Tasa de } \\
\text { mortalidad }\end{array}$ & $\begin{array}{c}27.3 \text { por } 100,000 \\
\text { habitantes }\end{array}$ & $\begin{array}{c}20.2 \text { por } 100,000 \\
\text { habitantes }\end{array}$ \\
\hline
\end{tabular}

$\mathrm{EPOC}=$ enfermedad pulmonar obstructiva crónica. 


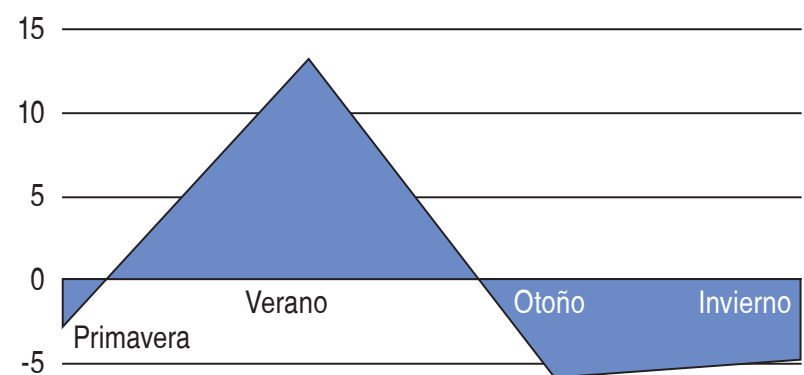

$-10$

Figura 1: Distribución estacional de muertes por EPOC en 2017 en Yucatán.

Mediante la prueba $\chi^{2}$ se comparó la proporción de pacientes que reciben asistencia médica previo a la defunción, comparando entre Yucatán y a nivel nacional ajustando por frecuencias; y se identificó una diferencia estadísticamente significativa ( $p=0.007)$, dado que a nivel nacional corresponde a $92.3 \%$ de los fallecimientos por EPOC, mientras que en el estado de Yucatán fue de 88.1\% (Figura 3).

\section{DISCUSIÓN}

Éste es el primer estudio que muestra un análisis epidemiológico de la mortalidad por EPOC en el estado de Yucatán; y las implicaciones de este incremento proporcional en el período invernal son de importancia para fines administrativos, asistenciales y diagnósticos.

Se identificó que la tasa de mortalidad de Yucatán fue mayor al compararla con la tasa a nivel nacional. Se expone que la tasa de mortalidad de México es menor al relacionarla con la de otros países en años anteriores. ${ }^{9-12}$

A nivel nacional la estación de verano se relaciona con menor porcentaje de muerte por EPOC. Sin embargo, en Yucatán se demostró que la estación de verano se relaciona con un aumento del número de muertos por EPOC, esto se contrapone a estudios, los cuales describen que la mortalidad de EPOC se encuentra ligada a estaciones y climas invernales. En lugares como Michigan, Nueva Zelanda y Taiwán se registró un incremento de la mortalidad por cada grado centígrado que baja la temperatura. El incremento de mortalidad por EPOC durante verano en el estado de Yucatán coincide con el comienzo de la temporada de lluvias de la región, además de que la variabilidad en la temperatura de la región permanece similar a lo largo de todo el año, razón por la cual los casos de muerte relacionados con EPOC no ocurren en invierno. ${ }^{13-15}$

Según Donaldson y Wedzicha existe una relación entre el aumento de las exacerbaciones y las variaciones estacionales, donde las regiones más cercanas a los hemisferios mostraban un aumento en el número de los casos en la temporada de invierno. Las causas a las cuales se ha asociado este incremento son el aumento de la temperatura, la cual provoca un incremento en la susceptibilidad de enfermarse por virus y bacterias estacionales, de igual modo la contaminación puede ser un factor que aunado a la temperatura detona las exacerbaciones. Otros factores que se deben tomar en consideración son el aumento de tabaquismo y el uso de biomasa como método de calefacción. ${ }^{16-18}$

Con relación al porcentaje de muertes en hombres y mujeres por EPOC, observamos que existe mayor número de muertes en hombres, lo cual se relaciona con otros estudios donde se puede observar que la mortalidad en hombres ha sido mayor a lo largo de los años; sin embargo, el número de muertes en hombres ha presentado una disminución mayor año con año en comparación con las mujeres. ${ }^{19-23}$

Se confirma que existe una diferencia significativa entre la edad a la que fallecieron las personas del sexo masculino por EPOC y las personas del sexo femenino, por lo que se infiere que los hombres tienen mayor probabilidad de fallecer a edades más tempranas que las mujeres, se apro-

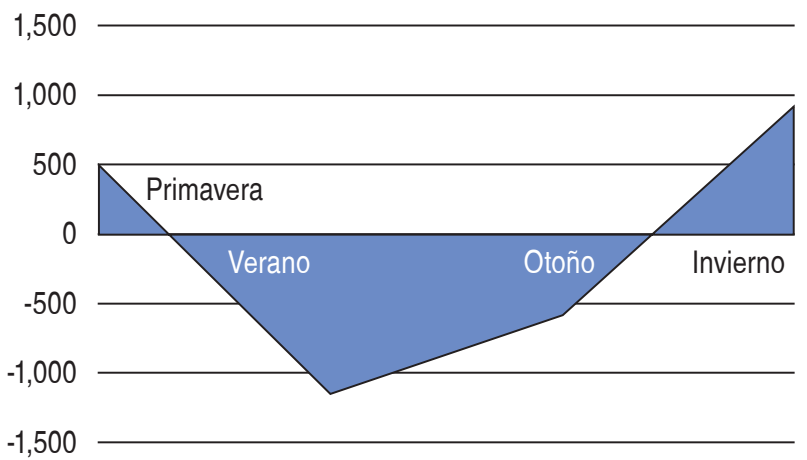

Figura 2: Distribución estacional de muertes por EPOC en 2017 a nivel nacional.

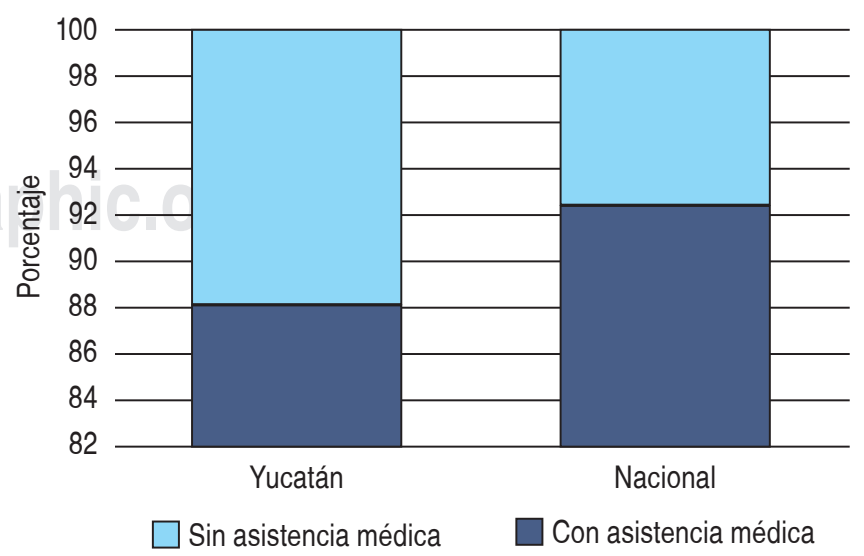

Figura 3: Porcentaje de muertos por EPOC que recibieron asistencia médica previa a su defunción en 2017. 
xima que la diferencia es alrededor de un año y tres meses menor en hombres. ${ }^{24-26}$

En relación al porcentaje de personas con EPOC que recibieron asistencia médica antes de su fallecimiento, se pudo comprobar que un menor número recibió dicho servicio en Yucatán al compararlo a nivel nacional. Esto se correlaciona con la estimación de la población con seguro de salud en México en cuanto a IMSS e ISSSTE. De igual forma, en el informe de salud de 2016 se menciona que Yucatán posee una disminución por debajo de la media en México en cuanto a consultorios por cada 10,000 habitantes por enditad federativa. ${ }^{27,28}$

También se puede correlacionar con un estudio en España donde la asistencia sanitaria por EPOC se ve disminuida en ciertos lugares de la nación, atribuidos a distintas variables de igual manera mencionadas en esta investigación.

La atención médica se puede correlacionar con los ingresos hospitalarios demostrados en estudios realizados según Tan y colaboradores donde se encontró que el acceso sanitario y hospitalario está relacionado al grado de urbanización de los pacientes y en cuyo estudio hubo una diferencia estadísticamente significativa en cuanto a diferentes regiones estudiadas como Australia y Canadá, y donde ciertas regiones presentaron una baja en cuanto hospitalizaciones y, por lo tanto, en asistencia médica. ${ }^{18,24,29-32}$

Por último, cabe recalcar que los datos obtenidos pueden mostrar variaciones debido a sesgos por parte del personal de salud al momento de registrar la causa de muerte.

\section{CONCLUSIÓN}

En el estado de Yucatán durante el año 2017 la mortalidad por EPOC fue menor durante la temporada invernal al compararse con las demás estaciones y contrastarse con las tendencias nacionales de variación en la mortalidad por EPOC.

\section{REFERENCIAS}

1. Chen AM, Bollmeier SG, Finnegan PM. Long-acting bronchodilator therapy for the treatment of chronic obstructive pulmonary disease. Ann Pharmacother 2008:42(12):1832-1842. https://doi.org/10.1345/ aph.1L250

2. Beall RF, Nickerson JW, Kaplan WA, Attaran A. Is patent "evergreening" restricting access to medicine/device combination products? PloS One 2016;11(2):e0148939. https://doi.org/10.1371/ journal.pone.0148939

3. Montserrat-Capdevila J, Godoy P, Ramon MJ, Barbé-Illa F. Factores asociados a la mortalidad global en los pacientes diagnosticados de enfermedad pulmonar obstructiva crónica. Aten Primaria 2015;47(8):498-504. http://dx.doi.org/10.1016/j.aprim.2014.11.004

4. Ramírez-Venegas A, Sansores RH, Pérez-Padilla R, et al. Survival of patients with chronic obstructive pulmonary disease due to biomass smoke and tobacco. Am J Resp Crit Care Med 2006;173(4):393-397. doi: 10.1164/rccm.200504-5680C

5. López M, Mongilardi N, Checkley W. Enfermedad pulmonar obstructiva crónica por exposición al humo de biomasa. Rev Perú Med Exp Salud Publica 2014;31(1):94-99. doi: https://doi.org/10.17843/ rpmesp.2014.311.14

6. Adeloye D, Chua S, Lee C, et al.; Global Health Epidemiology Reference Group (GHERG). Global and regional estimates of COPD prevalence: Systematic review and meta-analysis. J Global Health 2015;5(2):020415. https://doi.org/10.7189/jogh.05-020415

7. Solanes GI, Clarà PC. Causas de muerte y predicción de la mortalidad en EPOC. Arch Bronconeumol 2010;46(7):343-346. doi: 10.1016/j. arbres.2010.04.001

8. Wilkinson TMA, Aris E, Bourne S, et al. A prospective, observational cohort study of the seasonal dynamics of airway pathogens in the aetiology of exacerbations in COPD. Thorax 2017;72(10):919-927. doi: 10.1136/thoraxjnl-2016-209023

9. Aguilar-Vargas E, Azcorra H, Novelo-Pérez JP, Gómez-Carro S, Méndez-Domínguez N. Descripción de la variación estacional en la mortalidad materna de México y el estado de Yucatán 2002-2016. Ginecol Obstet Mex 2018;86(10):640-649. https://doi.org/10.24245/ gom.v86i10.2145

10. Mendez-Dominguez NI, Bobadilla-Rosado LO, Fajardo-Ruiz LS, Camara-Salazar A, Gomez-Carro S. Influenza in Yucatan in 2018: Chronology, characteristics and outcomes of ambulatory and hospitalized patients. Braz J Infect Dis 2019;23(5):358-362. doi: 10.1016/j.bjid.2019.08.009

11. Vazquez-Villagran LL, Laviada-Molina H, Prelip M, MendezDominguez N. On what to do where prodigal daughters might be increasing? Ann Hepatol 2019. pii:S1665-2681(19)32044-7. doi: 10.1016/j.aohep.2019.06.006

12. López-Campos JL, Tan W, Soriano JB. Global burden of COPD. Respirology 2016;21(1):14-23. https://doi.org/10.1111/resp.12660

13. Ford ES. Trends in mortality from COPD among adults in the United States. Chest 2015;148(4):962-970. doi: 10.1378/chest.14-2311

14. Wen $\mathrm{H}$, Xie $\mathrm{C}$, Wang $\mathrm{L}$, et al. Difference in long-term trends in COPD mortality between China and the U.S., 1992-2017: An age-periodcohort analysis. Int J Environ Res Public Health 2019;16(9):pii: E1529. doi: 10.3390/ijerph16091529

15. Berry CE, Wise RA. Mortality in COPD: causes, risk factors, and prevention. COPD 2010;7(5):375-382. doi: 10.3109/15412555.2010.510160

16. Hansel NN, McCormack MC, Kim V. The effects of air pollution and temperature on COPD. COPD 2016;13(3):372-379. doi: 10.3109/15412555.2015.1089846

17. Donaldson GC, Goldring JJ, Wedzicha JA. Influence of season on exacerbation characteristics in patients with COPD. Chest 2012;141(1):94-100. doi: 10.1378/chest.11-0281

18. Wise RA, Calverley PM, Carter K, Clerisme-Beaty E, Metzdorf N, Anzueto A. Seasonal variations in exacerbations and deaths in patients with COPD during the TIOSPIR ${ }^{\circledR}$ trial. Int J Chron Obstruct Pulmon Dis 2018;13:605-616. doi: 10.2147/COPD.S148393

19. Donaldson GC, Wedzicha JA. The causes and consequences of seasonal variation in COPD exacerbations. Int J Chron Obstruct Pulmon Dis 2014;9:1101-1110. doi: 10.2147/COPD.S54475

20. Chandra S, Chaloupka FJ. Seasonality in cigarette sales: patterns and implications for tobacco control. Tob Control 2003;12(1):105-107. doi: 10.1136/tc.12.1.105 
Neumol Cir Torax. 2020; 79 (1): 31-36

21. Kuri-Morales P, Alegre-Díaz J, Mata-Miranda MP, Hernández-Ávila M. Mortalidad atribuible al consumo de tabaco en México. Salud Publ Mex 2002;44(Supl 1):S29-S33.

22. Brehm JM, Celedón JC. Chronic obstructive pulmonary disease in Hispanics. Am J Respir Crit Care Med 2008;177(5):473-478. doi: 10.1164/rccm.200708-1274PP

23. Brown DW, Pleasants RA. Mortality from chronic obstructive pulmonary disease among adults aged 25 years or older in North Carolina. South Med J 2011;104(1):20-23. doi: 10.1097/ SMJ.0b013e3181fcda00

24. Tan WC, Seale P, Ip M, et al. Trends in COPD mortality and hospitalizations in countries and regions of Asia-Pacific. Respirology 2009;14(1):90-97. doi: 10.1111/j.1440-1843.2008.01415.x

25. Soriano JB, Maier WC, Egger $P$, et al. Recent trends in physician diagnosed COPD in women and men in the UK. Thorax 2000;55(9):789794. http://dx.doi.org/10.1136/thorax.55.9.789

26. Lindberg A, Jonsson AC, Rönmark E, Lundgren R, Larsson LG, Lundbäck $B$. Ten-year cumulative incidence of COPD and risk factors for incident disease in a symptomatic cohort. Chest 2005;127(5):15441552. doi: 10.1378/chest.127.5.1544

27. Gershon A, Hwee J, Victor JC, et al. Mortality trends in women and men with COPD in Ontario, Canada, 1996-2012. Thorax 2015;70(2):121-126. doi: 10.1136/thoraxjnl-2014-205956
28. Gershon AS, Wilton AS. Trends in chronic obstructive pulmonary disease prevalence, incidence, and mortality in Ontario, Canada, 1996 to 2007: a population-based study. Arch Intern Med 2010;170(6):560565. doi: 10.1001/archinternmed.2010.17

29. Hernández-Garduño E, Ocaña-Servín HL. Temporal trends in chronic obstructive pulmonary disease mortality in Mexico, 19992014. Int J Tuberc Lung Dis 2017;21(3):357-362. doi: 10.5588/ ijtld.16.0571

30. Puentes-Rosas E, Sesma S, Gómez-Dantés O. Estimación de la población con seguro de salud en México mediante una encuesta nacional. Salud Publ Mex 2005;47 supl 1:S22-S26.

31. Zamora RE, Alcántara RD, Cabrera SEG, Moreno MTC. Informe sobre la salud de los mexicanos 2016. México: Secretaría de Salud; 2016. Disponible en: http://www.gob.mx/salud/ acciones-y-programas/direccion-general-de-evaluacion-deldesempeno?idiom=es-MX

32. Jagana $\mathrm{R}$, Bartter $\mathrm{T}$, Joshi $\mathrm{M}$. Delay in diagnosis of chronic obstructive pulmonary disease: reasons and solutions. Curr Opin Pulm Med 2015;21(2):121-126. doi: 10.1097/ MCP. 0000000000000133

Conflicto de intereses: Los autores declaran no tener conflicto de intereses. 